\section{The impact of hepatitis C virus and human immunodeficiency virus coinfection on survival in patients with hepatocellular carcinoma}

\section{Eleftherios Spartalis ${ }^{a}$, Christos Damaskos ${ }^{b}$, Antonios Athanasiouc, Dimitrios Dimitroulis ${ }^{b}$}

University of Athens Medical School, Athens, Greece; Mercy University Hospital, Cork, Ireland

We read with great interest the article by Alkhalili et al [1], who concluded that, despite presenting with more advanced cirrhosis and being less likely to undergo surgery, patients with hepatocellular carcinoma (HCC) associated with viral hepatitis had similar survival to patients with $\mathrm{HCC}$ of non-hepatitis $\mathrm{B}$, non-hepatitis $\mathrm{C}$ etiology. But do these results reflect the whole picture?

Hepatitis $\mathrm{C}$ virus (HCV) and human immunodeficiency virus (HIV) share a common route of transmission, so that about one third of HIV infected individuals show HCV coinfection [2]. Highly active antiretroviral therapy has offered a longer and better life to infected patients. While this has removed HIV-related diseases from the list of most common causes of death, their place has been taken by complications of $\mathrm{HCV}$ infection, such as cirrhosis, end-stage liver disease, and HCC. HIV-HCV co-infection requires complex management, especially when HCC is present [2]. According to current guidelines, HCC treatment is the same for patients with and without HIV infection, although the outcome seems to be worse for HIV-positive patients than their HIV-negative counterparts [3].

The result of HCV infection is, in the majority of cases, the development of liver cirrhosis. Once cirrhosis is established, the annual risk of HCC, liver disease progression, and death in HCV-infected patients reaches approximately 1-7\%, 5\%, and $2 \%$, respectively [4]. HIV-HCV coinfected patients have a higher mean rate of fibrosis progression per year, translated into a shorter mean duration from HCV infection to cirrhosis, compared to HCV mono-infected patients [2]. Therefore, as described above, coinfected patients have been shown to develop liver cirrhosis more quickly than HCV-mono-infected individuals and demonstrate a more aggressive course of HCC [5], something that should have been mentioned in this study.

\section{References}

1. Alkhalili E, Greenbaum A, Luo L, et al. Viral hepatitis status does not affect survival in patients with hepatocellular carcinoma. Ann Gastroenterol 2017;30:101-105.

2. Dimitroulis D, Valsami S, Spartalis E, Pikoulis E, Kouraklis G. Hepatocellular carcinoma in patients co-infected with hepatitis $\mathrm{C}$ virus and human immunodeficiency virus. World J Hepatol 2013;5:323-327.

3. MacDonald DC, Nelson M, Bower M, Powles T. Hepatocellular carcinoma, human immunodeficiency virus and viral hepatitis in the HAART era. World J Gastroenterol 2008;14:1657-1663.

4. Singal AK, Anand BS. Management of hepatitis C virus infection in $\mathrm{HIV} / \mathrm{HCV}$ co-infected patients: clinical review. World $J$ Gastroenterol 2009;15:3713-3724.

5. Thomas DL. The challenge of hepatitis $\mathrm{C}$ in the HIV-infected person. Annu Rev Med 2008;59:473-485.

\footnotetext{
${ }^{a}$ Laboratory of Experimental Surgery and Surgical Research (Eleftherios Spartalis); bSecond Department of Propedeutic Surgery (Christos Damaskos, Dimitrios Dimitroulis), University of Athens Medical School, Athens, Greece; ' Department of Surgery, Mercy University Hospital, Cork, Ireland (Antonios Athanasiou)

Conflict of Interest: None
}

Correspondence to: Eleftherios Spartalis, Vasilissis Sofias 49, 10676 Athens, Greece, e-mail: eleftherios.spartalis@gmail.com

Received 16 February 2017; accepted 29 March 2017; published online 25 April 2017

DOI: https://doi.org/10.20524/aog.2017.0149 